\title{
Comprehensive characterization of cancer genes in hepatocellular carcinoma genomes
}

\author{
ZHIHAO ZHANG ${ }^{1 *}$, LIPING $\mathrm{XU}^{2 *}$ and CHANGYU SUN ${ }^{1}$ \\ ${ }^{1}$ Department of Infectious Diseases, The First Affiliated Hospital of Zhengzhou University, Zhengzhou, Henan 450052; \\ ${ }^{2}$ Department of Liver, Gallbladder and Pancreas Surgery, Ningbo First Hospital, Ningbo, Zhejiang 315010, P.R. China
}

Received February 1, 2017; Accepted October 20, 2017

DOI: 10.3892/ol.2017.7521

\begin{abstract}
The present study was performed to detect moderate or low-frequency mutated cancer driver genes in hepatocellular carcinoma (HCC), using OncodriveFM and Dendrix. Following this, integrated analyses were conducted on these novel cancer driver genes. A total of 112,980 somatic mutations were retrieved from TCGA and classified into 11 categories based on their function. Driver genes and pathways were predicted by OncodriveFM and Dendrix, followed by differential expression, DNA-methylation, copy number variations and survival analyses. Overall, non-synonymous mutations accounted for $>60 \%(72,149 / 112,980)$ of total variants, 108 and 3 driver genes were determined by OncodriveFM and Dendrix, respectively. Tumor protein $\mathrm{p} 53, \mathrm{SWI} / \mathrm{SNF}$ related, matrix associated, actin dependent regulator of chromatin, subfamily a, member 4 , smad family member 3 , RB transcriptional corepressor 1 , catenin $\beta 1$, smad family member 4 , mitogen-activated protein kinase 1 and TSC complex subunit 2 are at the core of the driver gene interaction network. Two genes, transportin 1 (TNPO1) and chaperonin containing TCP1 subunit 3 (CCT3), were hypomethylated and overexpressed, and high expression of TNPO1 and CCT3 indicated a poor prognosis in patients with HCC. $\beta$-carotene oxygenase 2 was hypermethylated, under-expressed and associated with favorable prognosis in HCC. The present study has identified a set of novel cancer genes and pathways, offering potential therapeutic targets and prognostic biomarkers for the treatment of HCC.
\end{abstract}

Correspondence to: Dr Changyu Sun, Department of Infectious Diseases, The First Affiliated Hospital of Zhengzhou University, 1 East Jianshe Road, Zhengzhou, Henan 450052, P.R. China

E-mail: scyuinfec@163.com

${ }^{*}$ Contributed equally

Key words: hepatocellular carcinoma, driver gene, driver pathway, copy number variation, survival

\section{Introduction}

Liver cancer is the fifth most prevalent type of cancer and the second most frequent cause of cancer-associated mortality in males, with an estimated 782,500 new cases and 745,500 deaths worldwide in 2012 (1). Hepatocellular carcinoma (HCC) is the most common type of liver cancer (2). HCC is particularly prevalent in East Asia, with an incidence rate twice that of Africa and $>4$ times higher than North America. However, the incidence rate of HCC has increased in Western countries (3). The dominant etiological cofactors that contribute to the incidence rate of HCC vary according to ancestry and region, including hepatitis B virus infection in East Asia and Africa, hepatitis C virus infection in Japan, aflatoxin B1 exposure in China and Africa and alcohol intake in Western countries $(4,5)$.

Mutational disruption of driver genes and pathways occurs constantly in cancers, enabling tumor cells to proliferate without constraints. A number of cancer genomics studies intend to prioritize driver genes based on recurrent mutation status across a cohort of tumor samples $(6,7)$. For instance, Totoki et al (8) applied MutSigCV to 503 pairs of HCC and matched non-cancerous liver tissues or blood and identified 30 recurrently mutated driver genes in HCC, including telomerase reverse transcriptase (TERT), catenin $\beta 1$ (CTNNB1), tumor protein p53 (TP53), AT-rich interaction domain 2 (ARID2) and axin 1 (AXINI). However, little attention has been paid to driver genes that are not frequently mutated, including genes that are enriched for mutations with high functional impact (FI) and genes with mutually exclusive mutation rates. Therefore, the development of computational tools that are able to detect moderate or low-frequency mutated driver genes is necessary to provide a more complete understanding of cancer genomics. Methods including Oncodrive-FM (9) aim to identify genes with a bias toward the enrichment of variants with a high FI, as measured by the Sorting Intolerant From Tolerant algorithm (10), PolyPhen2 (11) and Mutation Assessor (12). Another method, Dendrix (13), was constructed to uncover sets of genes which have at least one mutation in the majority of cancer samples. These bioinformatics tools, which complement existing methods, open novel avenues for the identification of potential driver genes involved in the tumorigenesis and progression of HCC. 
In the present study, our group assessed the moderate or low-frequency mutated driver genes predicted by Oncodrive-FM and Dendrix in HCC. In addition to the previously reported driver genes, our group identified novel cancer-driving genes and pathways, including potential treatment targets and prognostic biomarkers. Then, aberrant expression of these driver genes, DNA methylation levels, copy number variations (CNVs) and correlation with prognosis were assessed in patients with HCC. The present study highlights the importance of analyzing cancer-driving genes in an integrated fashion, and provides insights into the carcinogenesis and progression of HCC.

\section{Materials and methods}

Classification of cancer mutations. A total of 112,980 somatic mutations, comprised of 104,595 single-nucleotide variants (SNVs) and 8,385 small insertions or deletions (indels), were generated by whole-exome sequencing of 377 tumor tissues and paired normal tissues, and this data was downloaded from The Cancer Genome Atlas (TCGA; http://cancergenome.nih.gov/, accessed on January 15, 2016) (14). Somatic mutations were classified into 11 categories based on their functional impacts in the coding genome using Ensembl Variant Effect Predictor (VEP) (15).

Prediction of driver genes and pathways. Driver genes and pathways were determined using OncodriveFM(https://www. intogen.org/analysis) and Dendrix (http://compbio.cs.brown. edu/projects/dendrix/) with default parameters following the criteria that genes and pathways have q values smaller than 0.05 . To clarify the functional enrichment of driver genes, Gene Ontology (GO) terms $(16,17)$ and Kyoto Encyclopedia of Genes and Genomes (KEGG) (18) pathway enrichment analyses were conducted for all the driver genes on the home page of STRING (http://string.embl.de/) (19). The GO terms and KEGG pathways were considered to be significantly enriched for driver genes with the cut-off of a false positive rate of $\mathrm{P}<0.05$. Finally, a protein-protein interaction network was constructed with STRING to prioritize the pivotal driver genes in HCC. For each driver gene, the overall STRING score that represents the degree to which the driver gene is associated with other genes was computed by summing up combined STRING scores of all its protein-protein interactions.

Gene expression, DNA methylation and principal component analyses. The present study used expression data from Gao et al (20), which were generated by RNA sequencing of 8 paired HCC and normal tissues, and deposited in the Gene Expression Omnibus database (21) (accession no. GSE55758). DNA methylation data from 340 HCC samples were obtained from TCGA (14), and unavailable values were replaced with the mean $\beta$ value. Differentially expressed and dysmethylated genes between HCC and normal tissues were determined using the function of t-test in $\mathrm{R}$ with the cutoff value of $\mathrm{P}<0.05$. Next, principal component analysis (PCA) was conducted using the princomp function in $\mathrm{R}$ to examine whether the differentially expressed genes distinguished cancer tissues from normal tissues.

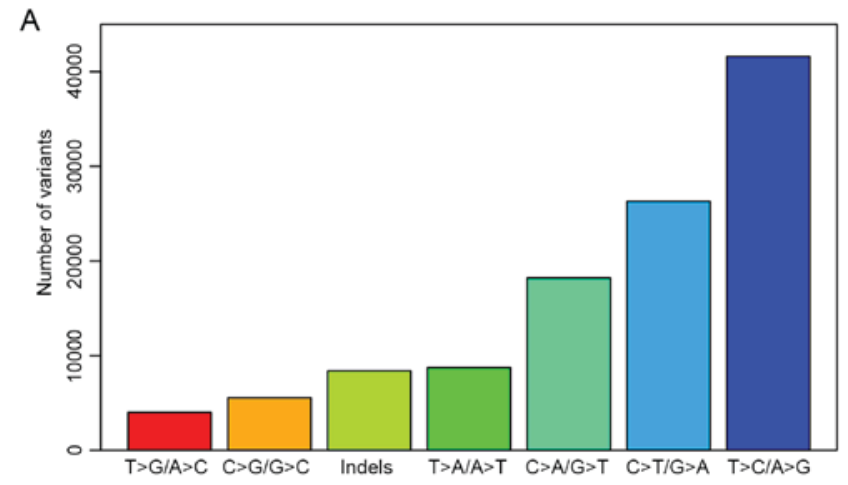

B

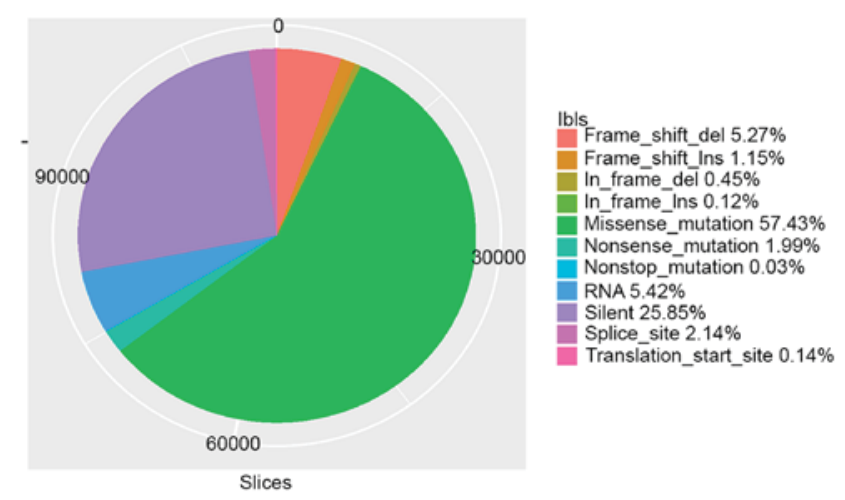

Figure 1. Catalog of somatic mutations. (A) Mutation signatures in HCC. (B) The number and proportion of 11 groups of somatic mutations in HCC. $\mathrm{HCC}$, hepatocellular carcinoma; indel, insertions or deletions.

Sources of CNV and survival analyses. CNVs in $370 \mathrm{HCC}$ samples, detected by single nucleotide polymorphism (SNP) array, were acquired from the Broad Institute TCGA GDAC Firehose (gdac.broadinstitute.org) (14). RNA sequencing (RNAseq) and clinical outcome data were retrieved from TCGA to further evaluate whether the expression of driver genes was associated with prognosis in $360 / 377$ patients with HCC (14). For each driver gene, the patients with HCC were divided into high and low expression groups. The former refers to $25 \%(n=90)$ of patients with the highest RNA expression levels of the driver gene, while the latter refers to the $25 \%(n=90)$ of patients with the lowest expression levels of driver gene. Kaplan-Meier survival analyses were performed between the high and low expression groups using oncolnc (http://www.oncolnc.org/) (22) and the log-rank test was utilized to compare the difference of survival rates between different groups. $\mathrm{P}<0.05$ was considered to indicate a statistically significant difference.

\section{Results}

Catalog of somatic mutations. A total of 112,980 somatic mutations generated by the whole-exome sequencing of 377 paired HCC and normal samples were downloaded from TCGA. Among them, 104,595, 8,385 mutations were SNVs and small indels, respectively. $\mathrm{T}>\mathrm{C} / \mathrm{A}>\mathrm{G}, \mathrm{C}>\mathrm{T} / \mathrm{G}>\mathrm{A}$ and $\mathrm{C}>\mathrm{A} / \mathrm{G}>\mathrm{T}$ were the three most prevalent transitions in $\mathrm{HCC}$, with mutation rates of 36.82, 23.29 and $16.13 \%$ in all variant types (Fig. 1A). There were 64,890 missense mutations, 2,250 nonsense 


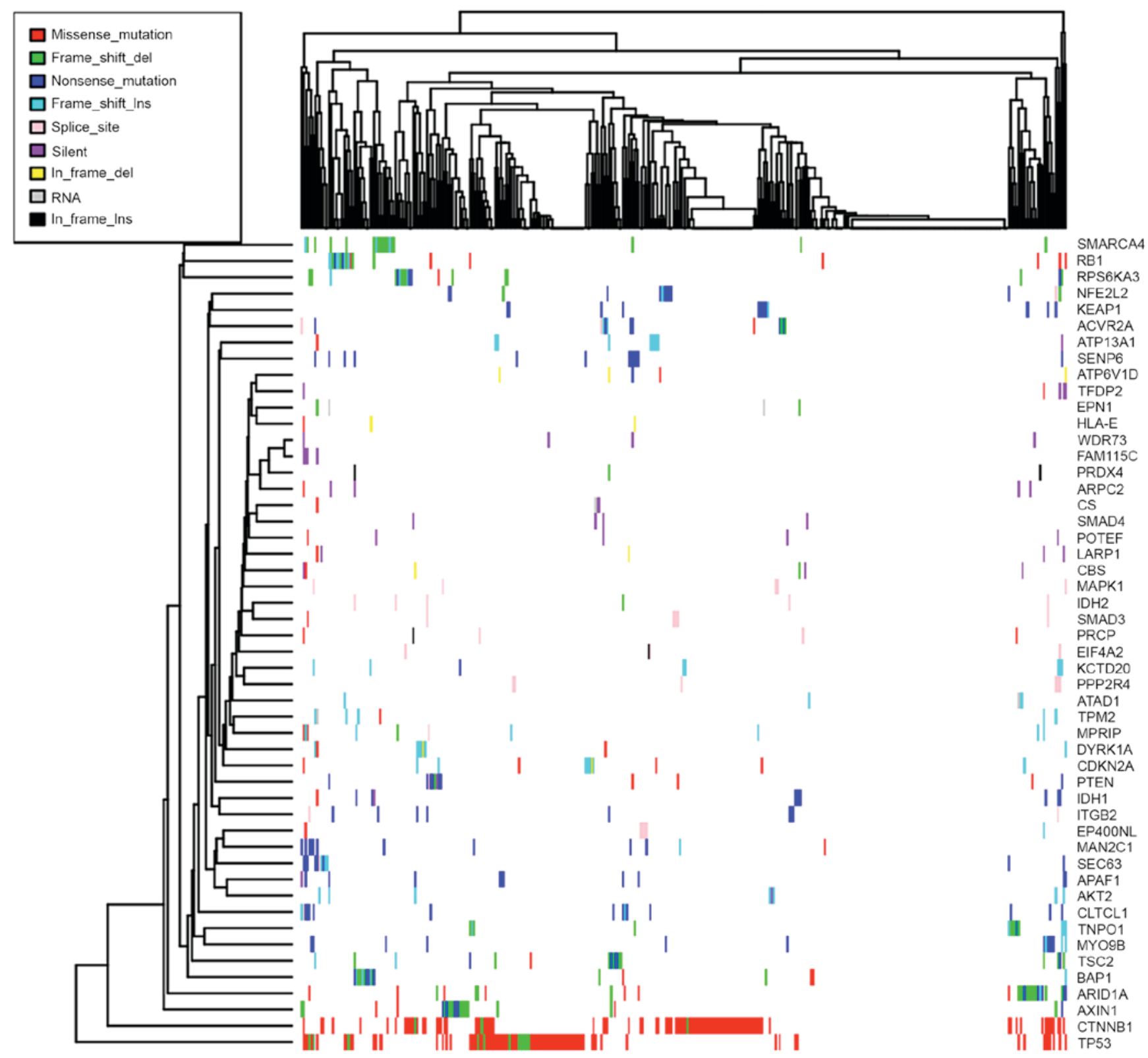

Figure 2. Mutation patterns of 50 cancer driver genes across 377 hepatocellular carcinoma samples.

mutations, 34 nonstop mutations and 29,209 silent SNVs classified by VEP. In total, 505 deletions and 135 insertions caused translational frame shifts, while 5,957 deletions and 1,302 insertions were in frame mutations. A total of 2,419 and 155 mutations occurred in splicing sites and translation start sites, respectively. Over $60 \%(72,149 / 112,980)$ of total variants were non-synonymous mutations (Fig. 1B). HCC has a lower nonsynonymous mutation density (2.03 nonsynonymous mutations per $\mathrm{Mb}$ per sample, on average) in comparison with other types of cancer, including melanoma and lung cancer (6).

Cancer driver genes and pathways in HCC. OncodriveFM was used to identify driver genes and pathways in HCC. In total, 108 driver genes were identified by OncodriveFM. Among them, TP53, CTNNB1, ARID2, AXIN1 and TERT were known driver genes, with mutation rates of 30.69, 27.11, $11.00,7.16$ and $2.05 \%$ across all HCC samples (8). However, the majority of driver genes were middle or low-frequency mutated genes, Fig. 2 presents the mutation patterns of the 50 most frequently mutated driver genes, however data are not shown for the remaining driver genes. Dendrix analysis was performed for sets of size ranging from 2 to 4 . When $\mathrm{k}=2$, the pair TP53 and CTNNB1 was sampled with a frequency of $24 \%$ $(240 / 1,000)$. When $\mathrm{k}=3$, the triplet [BRCA associated protein 1 (BAP1), TP53 and CTNNB1] was sampled with a frequency of $5.4 \%(54 / 1,000)$. For $\mathrm{k}=4$, no gene set had a sample frequency $>1 \%$. The pair (TP53 and CTNNB1) and triplet (BAP1, TP53 and $C T N N B 1$ ) were the most prevalent gene sets in the mutual exclusivity test, further supporting the importance of TP53 and CTNNB1 in the tumorigenesis of HCC.

The enrichment of GO terms was performed for 109 cancer genes on STRING, and 80 biological processes, 36 molecular functions and 47 cellular components were reported with statistical significance. The GO terms represented a wide variety of functional processes, including 'regulation of protein metabolic process', 'regulation of cellular process', 'regulation of gene 


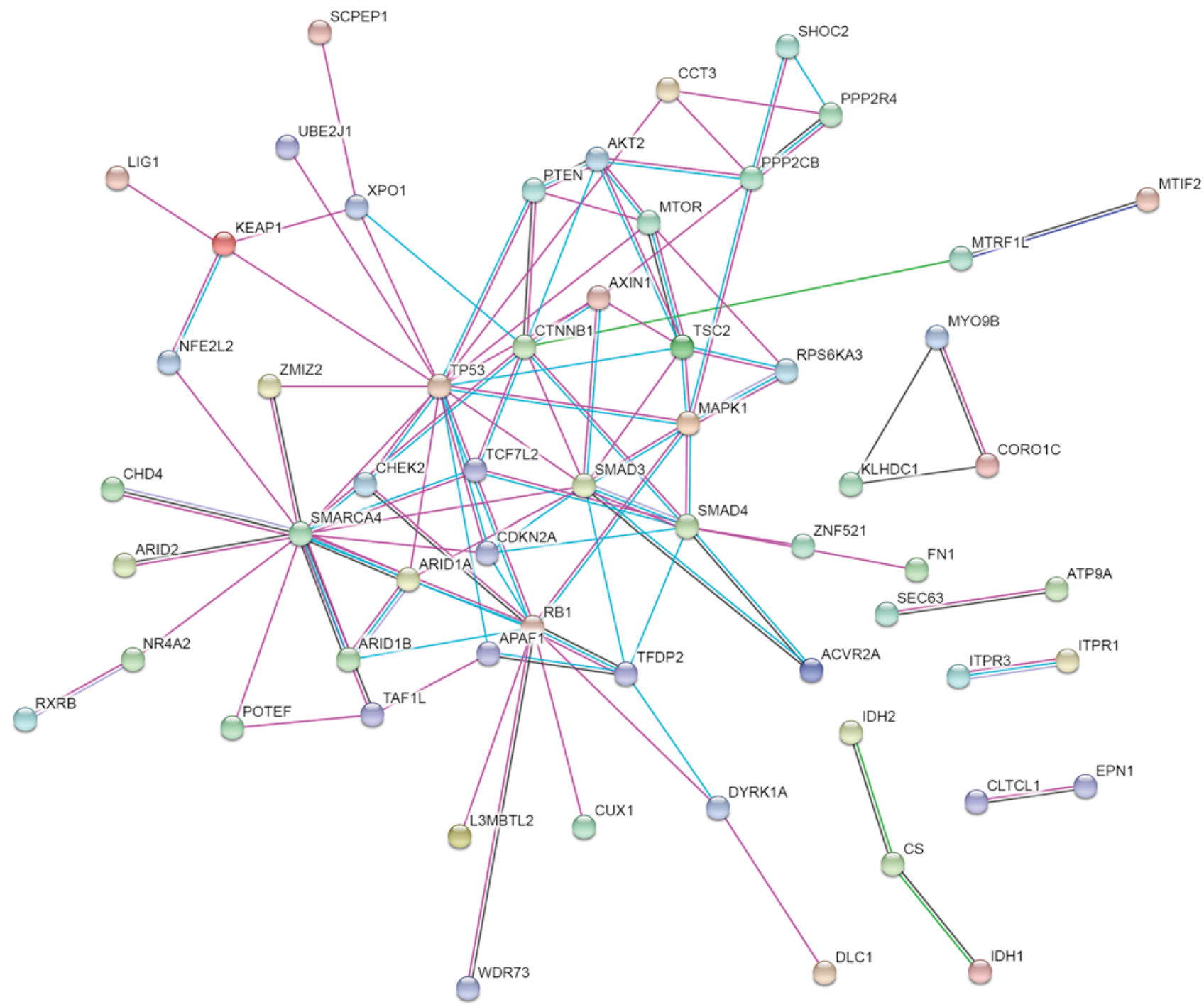

Figure 3. Protein-protein interaction network. Network nodes represent proteins, edges represent protein-protein associations. Disconnected nodes were disabled in the network. Red nodes refer to query proteins and the first shell of interactors, white nodes are the second shell of interactors. Light blue lines represent known interactions from the curated Kyoto Encyclopedia of Genes and Genomes database, purple lines represent experimentally determined protein-protein interactions, green lines denote genes that are frequently observed in each other's genomic neighborhood, black lines stand for genes where expression are correlated across a large number of experiments, dark blue lines indicate gene families with similar occurrence patterns across genomes and light grey lines indicate proteins with amino acid sequence similarity.

expression', 'cell cycle' and 'cellular component biogenesis and organization', indicating that driver gene candidates are actively involved in cancer-associated processes in HCC (data not shown). The 109 driver genes were significantly enriched in 58 KEGG pathways, including 'cell cycle', 'p53 signaling pathway', 'mTOR signaling pathway' and 'PI3K-Akt signaling pathway' (data not shown). In addition, Oncodrive-FM also identified 119 pathways with statistical FI bias in HCC, including 'colorectal cancer', 'endometrial cancer', 'basal cell carcinoma', 'thyroid cancer', 'prostate cancer', 'glioma', 'wnt signaling pathway', 'pathways in cancer', 'p53 signaling pathway', 'pancreatic cancer', 'non-small cell lung cancer' and 'melanoma' (data not shown). A total of 71.19\% (42/59) of driver gene-enriched pathways were overlapped with driver pathways in HCC. Finally, a protein-protein interaction network was constructed with STRING to prioritize the pivotal driver genes. As presented in Fig. 3, TP53, SWI/SNF related, matrix associated, actin dependent regulator of chromatin, subfamily a, member 4 (SMARCA4), smad family member 3, RB transcriptional corepressor 1 (RB1), CTNNB1, smad family member 4 (SMAD4), mitogen-activated protein kinase 1 (MAPKI) and TSC complex subunit 2 are at the core of the protein-protein interaction network. This suggests that they may serve key functions in the network, because they possessed the strongest protein-protein interactions (overall STRING score $>5$; data not shown).

Expression profiling and DNA methylation analyses in HCC. To analyze the gene expression profile in HCC, RNA-seq data from 8 paired $\mathrm{HCC}$ and normal tissues were obtained. Overall, 4,665 differentially expressed genes were identified between the 8 paired HCC and normal samples (Fig. 4A), of which 34 were driver gene candidates, including transportin 1 (TNPO1), apoptotic peptidase activating factor 1 (APAF1), AT-rich interaction domain 1A (ARIDIA) and BAPI (Fig. 4B). PCA was applied to examine whether differentially expressed genes 
A

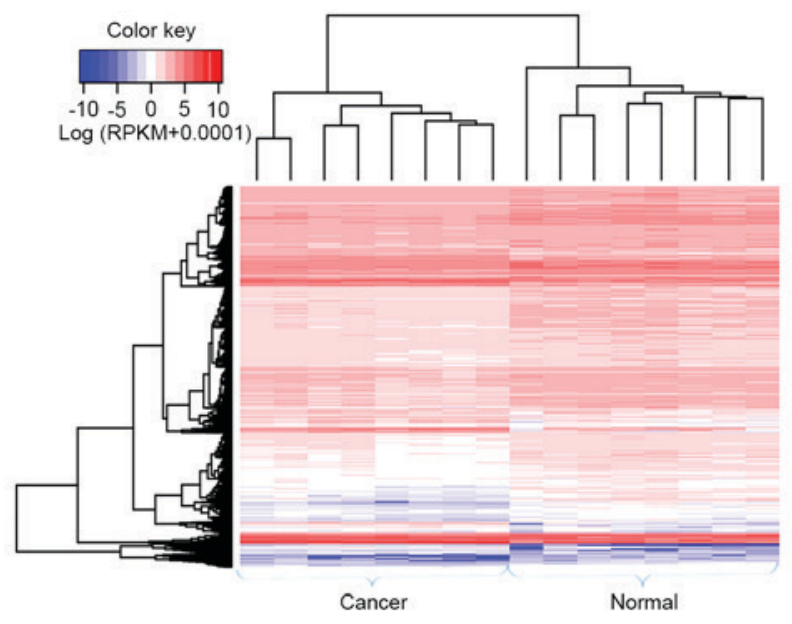

C

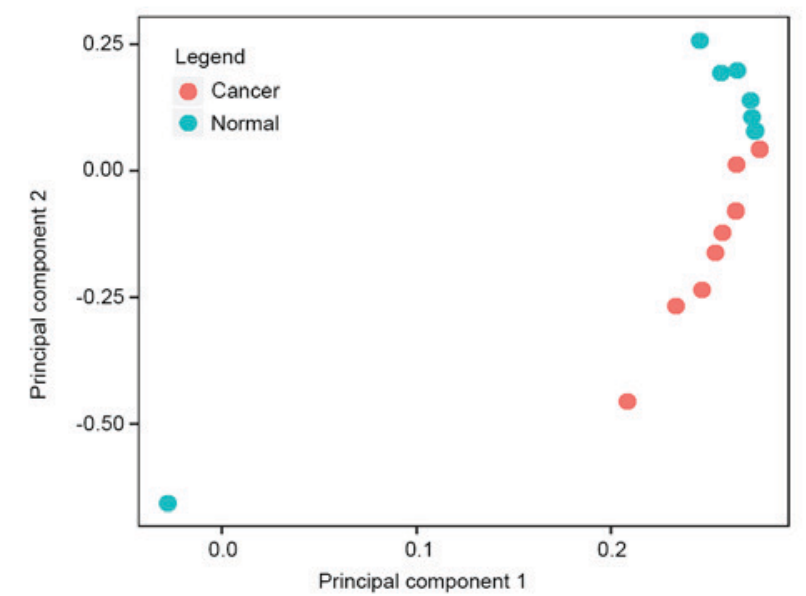

B

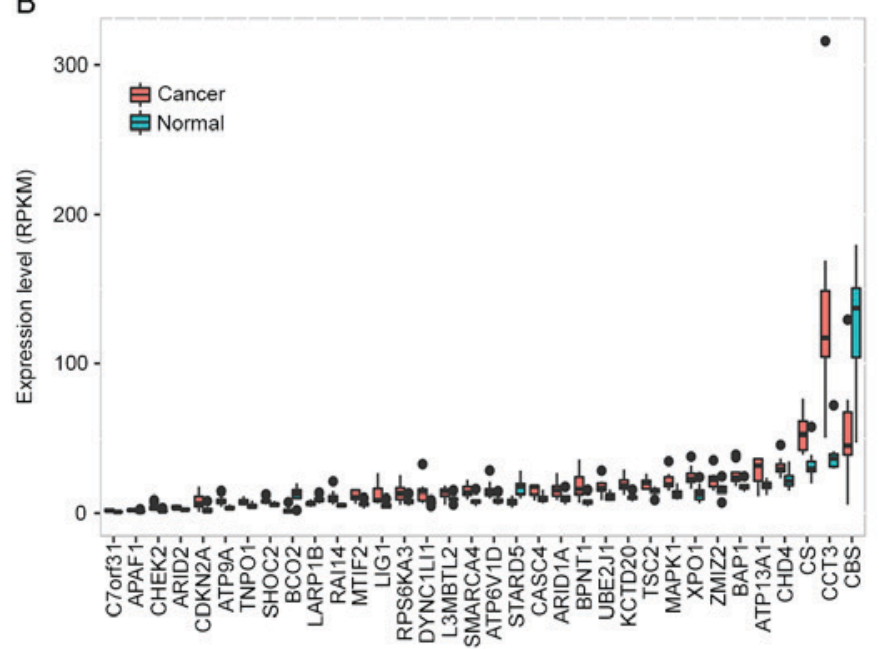

D

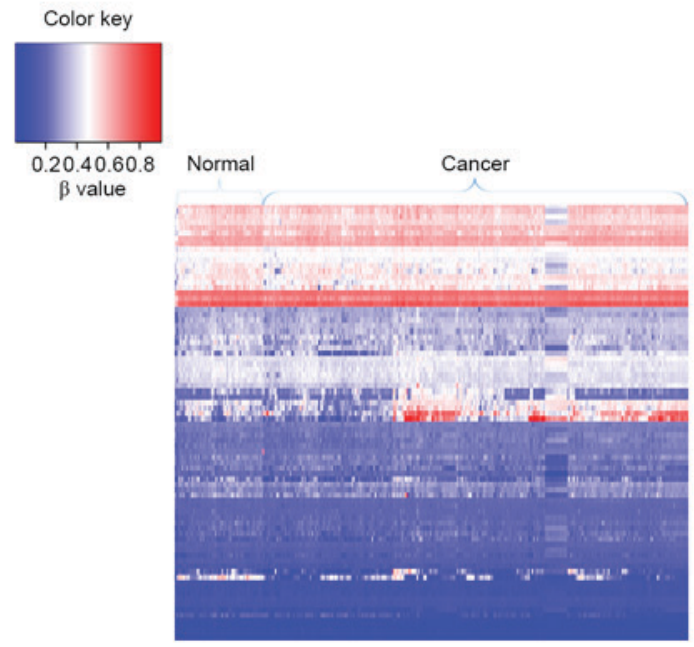

Figure 4. Expression profiling and DNA methylation analyses in HCC. (A) Expression clustering of 4,665 differentially expressed genes between HCC tissues and normal samples. The expression of each gene was presented as reads per kilobase of transcript per million mapped reads plus 0.0001 and log scaled. Red and blue represent high and low expression levels of differentially expressed genes, respectively. (B) The differential expression of 34 driver gene candidates between HCC and normal tissues. (C) Principle component analysis of the 8 paired cancer tissues and normal tissues using 4,665 differentially expressed genes. Red and blue dots denote cancer and normal tissues, respectively. Each dot represents the expression values of the significant genes that were summarized at the first two principal component coordinates. (D) DNA methylation profiling of 86 dysmethylated driver gene candidates. The average $\beta$ value was calculated for each gene and patient, and undefined values were replaced with the mean. HCC, hepatocellular carcinoma. Red and blue represent high and low DNA methylation values of driver genes, respectively.

were able to discriminate cancer tissues from normal tissues. As presented in Fig. 4C, normal tissues were aggregated to the upper side of the graph, whereas cancer tissues were clustered to the lower side, suggesting that differentially expressed genes were able to separate the tissue samples into two distinct groups.

DNA methylation of cytosine within $\mathrm{CpG}$ dinucleotides maintains the proper regulation of gene expression and stable gene silencing; therefore, DNA methylation alterations make a substantial contribution to tumorigenesis (23). In order to characterize the DNA methylation status of driver gene candidates, our group downloaded DNA methylation data from TCGA and analyzed the DNA methylation levels of the identified driver genes in HCC. Overall, 83 driver gene candidates had dysmethylation at their promoters, including 45 hypomethylated and 38 hypermethylated driver genes (Fig. 4D). These results suggest that the majority of driver genes may be implicated in HCC through modulating methylation status. 13 driver genes are overexpressed and hypomethylated, including chaperonin containing TCP1 subunit 3 (CCT3), checkpoint kinase 2 (CHEK2), SMARCA4, MAPK1, TNPO1 and mitochondrial translational initiation factor 2, suggesting they may have oncogenic functions in HCC. In contrast, $\beta$-carotene oxygenase 2 (BCO2) and cystathionine- $\beta$-synthase $(C B S)$ exhibited hypermethylation and low expression, and may therefore function as tumor suppressors in HCC.

CNVs in HCC. CNVs of 370 HCC samples detected by SNParray were also obtained from the Broad Institute. Significant focal gains and deletions $(\mathrm{q}<0.25)$ were identified in 360 samples (360/370, 97.30\%) at 61 loci (34 amplifications and 27 deletions). Among these, amplifications at 1q22 and 1q42.3 and deletions at $8 \mathrm{p} 23.2$ and $8 \mathrm{p} 12$ were the most frequent CNVs in HCC, with occurrence rates of $76.49 \%$ (90/370), $72.97 \%$ 


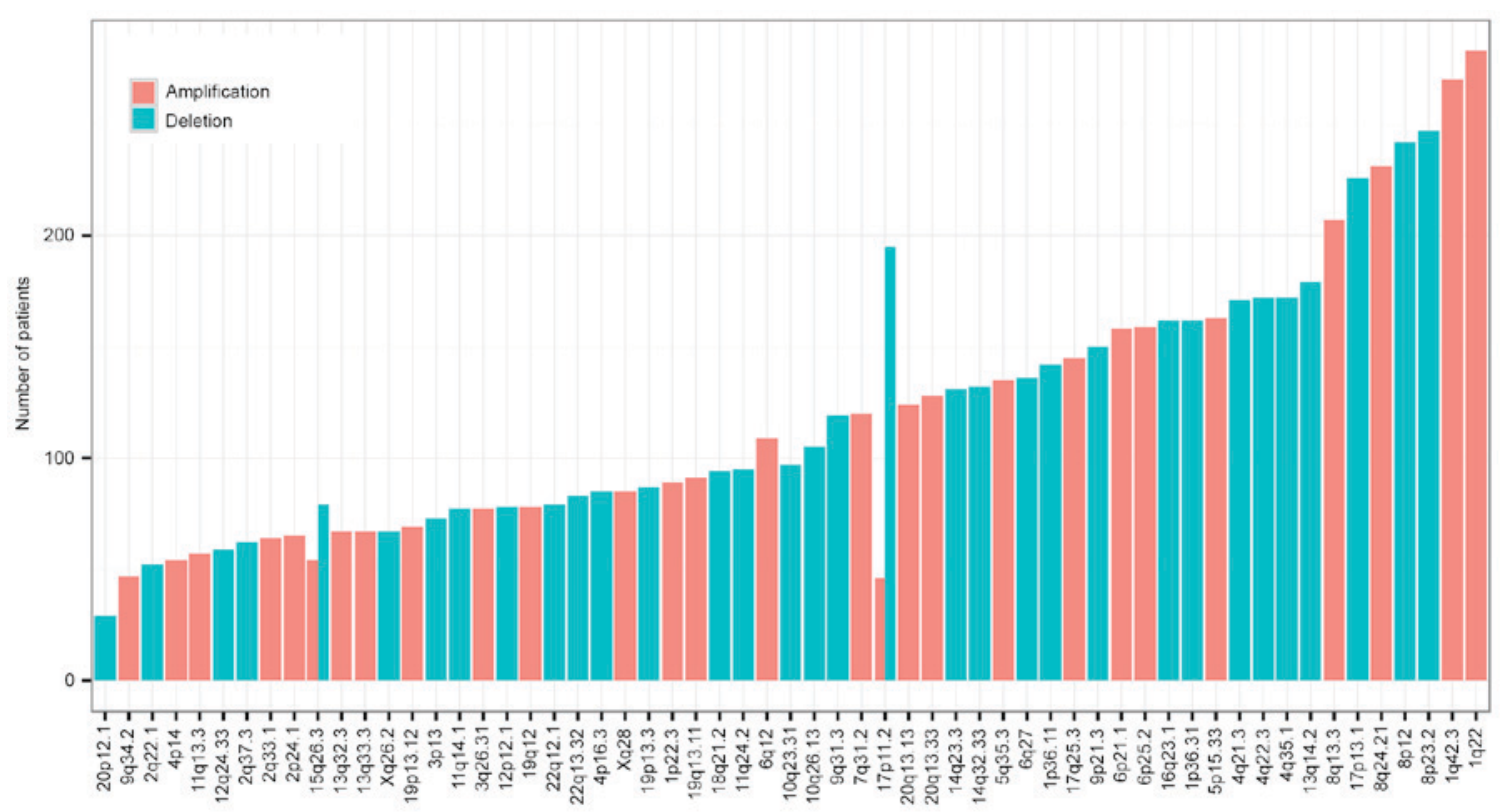

Figure 5. Frequencies of copy number variations in 370 hepatocellular carcinoma samples.

(89/370), 66.76\% (89/370) and 65.41\% (90/370), respectively (Fig. 5). A total of 17 cancer driver genes were involved in CNVs, including known tumor suppressors and oncogenes, for example TP53 (deletion, 17p13.1), phosphatase and tensin homolog (deletion, 10q23.31) and $R B 1$ (deletion 13q14.2). Multiple driver candidates were also implicated in the CNVs, including SHOC2, leucine rich repeat scaffold protein (deletion, 10q25.2), transcription factor 7 like 2 (TCF7L2; deletion, 10q25.2), SMAD4 (deletion, 18q21.2), TRPM8 channel associated factor 2 (deletion, 7q35), isocitrate dehydrogenase [NADP (+2)] mitochondrial $(I D H 2)$ and DNA polymerase $\gamma$, catalytic subunit (deletion, 15q26.1), solute carrier family 2 member 6 (deletion, 9q34.2), outer dense fiber of sperm tails 2 and protein phosphatase 2 phosphatase activator (deletion, 9q34.11), ARID1A (deletion, 1p36.11), cut like homeobox 1 (deletion, 7q22.1), cyclin dependent kinase inhibitor 2A (CDKN2A; deletion, 9p21.3), BCO2 (deletion, 11q23.1) and EP400 N-terminal like (deletion, 12q24.33).

Survival analyses. TCGA RNAseq and clinical outcome data were acquired from TCGA to evaluate whether the expression of 109 driver genes is associated with survival and prognosis in patients with HCC. Kaplan-Meier survival analysis showed that the expression of 24 driver genes was significantly associated with clinical outcomes in patients with HCC. High expression of 14 driver genes indicated worse survival rates in patients with $\mathrm{HCC}$, including $A X I N I$, serine carboxypeptidase 1, APAF1, TRPM8 channel associated factor 2, actin related protein $2 / 3$ complex subunit 2 , autophagy related $9 \mathrm{~A}$, exportin 1 (XPO1), Basic Leucine Zipper and W2 domains 2, CCT3, CDKN2A, chromodomain helicase DNA binding protein 4 , coronin $1 \mathrm{C}, \mathrm{RAB} 32$, member RAS oncogene family and TNPO1. Fig. 6A presents the correlation between the expression of $X P O 1$ and patients' clinical outcome; data are not shown for the remaining driver genes (Fig. 6A). In contrast, patients with high expression of 10 other driver genes had a relatively favorable prognosis, including kinesin 2, $\mathrm{BCO} 2$,
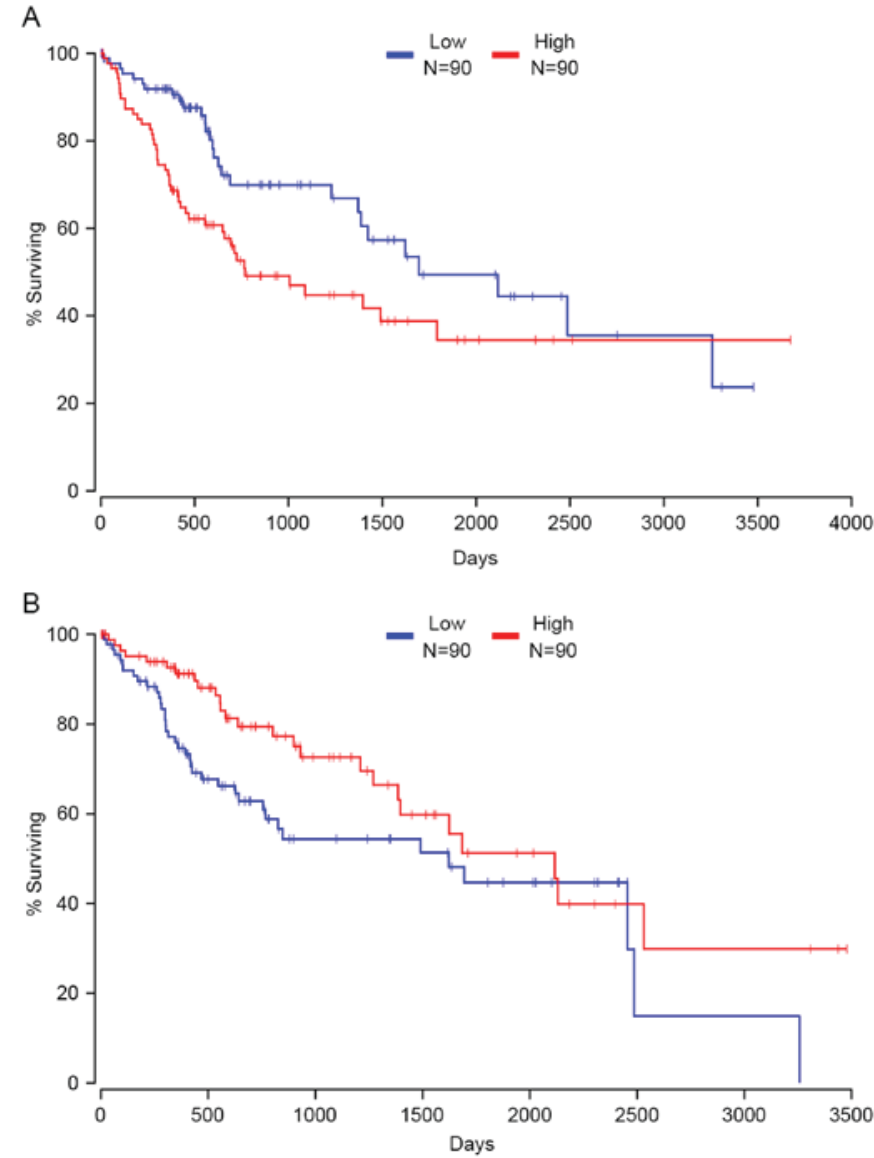

Figure 6. Survival analyses. (A) Patients with high expression of XPOI (red) had a relatively poor survival rate compared with those with low expression of $X P O 1$ (blue). (B) Patients with high expression of $I D H 2$ (red) had an improved prognosis compared with those with low expression of $I D H 2$ (blue). XPO1, exportin 1; IDH2, isocitrate dehydrogenase [NADP (+2)] mitochondrial.

cancer susceptibility 4, GTF2I repeat domain containing 2B, major histocompatibility complex, class I, E, IDH2, 
La ribonucleoprotein domain family member $1 \mathrm{~B}$, mannosidase alpha class 2C member 1, zinc finger protein 521 and StAR related lipid transfer domain containing 5 (data not shown). TNPO1 and CCT3 were hypomethylated, overexpressed and associated with a poor prognosis in HCC. BCO2 was hypermethylated, under-expressed and associated with a favorable prognosis in HCC. These three driver genes may be potential targets for treatment and prognostic biomarkers for patients with $\mathrm{HCC}$ in the future.

\section{Discussion}

In the present study, our group conducted an integrated investigation of 109 cancer-driving genes and 119 pathways determined with Oncodrive-FM and Dendrix. Only a small fraction of these driver genes are repeatedly mutated in HCC samples, including TP53, CTNNB1, ARID2, AXIN1 and TERT (8). The P53-retinoblastoma (RB) signaling pathway was consistently mutated in cancer samples, including TP53 and $C H E K 2$ of the P53 pathway, and RB1 and CDKN2A of the RB pathway, reflecting the inactivation of the P53-RB pathway in HCC. In addition to those identified in the P53-RB pathway, inactivating mutations were frequently observed in WNT pathway genes, including AXIN1 and CTNNB1. Multiple cancer driver genes initially identified in other types of cancer were first identified as drivers in $\mathrm{HCC}$, including $B A P 1$ in renal cell carcinoma (24), $I D H 2$ in angioimmunoblastic T-cell lymphoma (25), CDKN2A in melanoma (26), TCF7L2 in colorectal cancer (27) and SMAD4 in colorectal and pancreatic cancer (28). In addition, multiple novel driver candidates were identified by our group; for instance, $L A R P 1$ and $A P A F 1$. LARP1, as a conserved RNA-binding protein, interacts with poly-A-binding protein and modulates 5'-terminal oligopyrimidine tract mRNA translation. Enhanced expression of LARP1 increases cell migration, invasion, growth and tumorigenicity in vivo through post-transcriptionally altering gene expression, including mechanistic target of rapamycin, in Hela cells (29). APAF1 is an important factor that regulates the mitochondrial apoptotic pathway, and loss of APAF1 causes cellular resistance against apoptotic signals. Reduced expression of APAF1 has been observed in colorectal adenocarcinoma and is associated with deeper tumor invasion, frequent lymph node metastasis, higher stage and poorer differentiation (30). In addition, $A P A F 1$ is frequently methylated in bladder and kidney cancer, and demethylation of APAF1 increased APAF1 expression and inhibited the proliferation of bladder and kidney cancer cell lines (31).

One notable strength of OncodriveFM and Dendrix analysis lies in the identification of cancer-associated genes and pathways which have a high FI bias towards accumulating high FI variants or mutational exclusivity, independent of cancer mutation frequency. Implementing these tools allows for a comprehensive exploration of cancer-driving genes and pathways. In addition, our group revealed 35 differentially expressed genes, 83 dysmethylated driver genes and 17 cancer driver genes involved in CNVs, suggesting these genes may contribute to the development and progression of $\mathrm{HCC}$ in various ways.

Of the 109 driver gene candidates, 24 genes whose expression levels were associated with HCC patient prognosis were identified, including $X P O 1$ and $I D H 2$. $X P O 1$, also known as CRM1, encodes a protein which serves a critical function in the trafficking of over 230 proteins, including tumor suppressors (for example, p53, p73 and forkhead box O1), growth regulator/pro-inflammatory (for example, I $\mathrm{B}, \mathrm{Rb}$, p21, p27, BRCA1, DNA repair associated and APC, WNT signaling pathway regulator), and anti-apoptotic proteins (for example, nucleophosmin 1 and AP-1) (32). XPO1 is an oncogenic, anti-apoptotic protein in transformed cells and is overexpressed in a number of types of cancer, including mantle cell lymphoma (33), lung adenocarcinoma (34) and gastric cancer (35). Similar to the results of the present study, high expression of XPO1 is an independent poor prognostic factor in gastric carcinoma (35), acute myeloid leukemia (36), pancreatic cancer (37) and lung adenocarcinoma (34). Another gene, $I D H 2$, is frequently mutated in multiple types of cancer, including T-cell lymphoma (38), glioma (39) and acute myeloid leukemia (40). In line with the results of the present study, Liu et al (41) reported that decreased expression of IDH2 was associated with lower overall survival rates in HCC. Furthermore, mutated IDH2 was an independent prognostic factor for improved overall survival in acute myeloid leukemia (40) and glioma (42). Therefore, these genes may be ideal candidates for the development of HCC prognostic indicators in future studies.

In summary, our group have successfully compiled a list of cancer driver genes and pathways in HCC, enhancing our understanding of pathogenesis and progression of HCC. Furthermore, the novel driver genes and pathways identified by the present study pave the way for the development of therapies targeting driver genes and prognostic biomarkers in HCC.

\section{References}

1. Torre LA, Bray F, Siegel RL, Ferlay J, Lortet-tieulent J and Jemal A: Global cancer statistics, 2012. CA Cancer J Clin 65: 87-108, 2015

2. Massarweh NN and El-Serag HB: Epidemiology of hepatocellular carcinoma and intrahepatic cholangiocarcinoma. Cancer Control 24: 1073274817729245, 2017.

3. Center MM and Jemal A: International trends in liver cancer incidence rates. Cancer Epidemiol Biomarkers Prev 20: 2362-2368, 2011

4. Yu J, Shen J, Sun TT, Zhang X and Wong N: Obesity, insulin resistance, NASH and hepatocellular carcinoma. Semin Cancer Biol 23: 483-491, 2013.

5. Augustine MM and Fong Y: Epidemiology and risk factors of biliary tract and primary liver tumors. Surg Oncol Clin N Am 23: 177-188, 2014.

6. Lawrence MS, Stojanov P, Polak P, Kryukov GV, Cibulskis K, Sivachenko A, Carter SL, Stewart C, Mermel CH, Roberts SA, et al: Mutational heterogeneity in cancer and the search for new cancer-associated genes. Nature 499: 214-218, 2013.

7. Dees ND, Zhang Q, Kandoth C, Wendl MC, Schierding W, Koboldt DC, Mooney TB, Callaway MB, Dooling D, Mardis ER, et al: MuSiC: Identifying mutational significance in cancer genomes. Genome Res 22: 1589-1598, 2012.

8. Totoki Y, Tatsuno K, Covington KR, Ueda H, Creighton CJ, Kato M, Tsuji S, Donehower LA, Slagle BL, Nakamura H, et al: Trans-ancestry mutational landscape of hepatocellular carcinoma genomes. Nat Genet 46: 1267-1273, 2014.

9. Gonzalez-Perez A and Lopez-Bigas N: Functional impact bias reveals cancer drivers. Nucleic Acids Res 40: e169, 2012.

10. Sim NL, Kumar P, Hu J, Henikoff S, Schneider G and Ng PC: SIFT web server: Predicting effects of amino acid substitutions on proteins. Nucleic Acids Res 40 (Web Server Issue): W452-W457, 2012. 
11. Adzhubei IA, Schmidt S, Peshkin L, Ramensky VE, Gerasimova A, Bork P, Kondrashov AS and Sunyaev SR: A method and server for predicting damaging missense mutations. Nat Methods 7: 248-249, 2010.

12. Reva B, Antipin Y and Sander C: Predicting the functional impact of protein mutations: Application to cancer genomics. Nucleic Acids Res 39: e118, 2011.

13. Vandin F, Upfal E and Raphael BJ: De novo discovery of mutated driver pathways in cancer. Genome Res 22: 375-385, 2012.

14. Cancer Genome Atlas Research Network. Electronic address: wheeler@bcm.edu; Cancer Genome Atlas Research Network: Comprehensive and integrative genomic characterization of hepatocellular carcinoma. Cell 169: 1327-1341.e23, 2017.

15. McLaren W, Pritchard B, Rios D, Chen Y, Flicek P and Cunningham F: Deriving the consequences of genomic variants with the ensembl API and SNP effect predictor. Bioinformatics 26: 2069-2070, 2010.

16. Ashburner M, Ball CA, Blake JA, Botstein D, Butler H, Cherry JM, Davis AP, Dolinski K, Dwight SS, Eppig JT, et al: Gene ontology: Tool for the unification of biology. The Gene Ontology Consortium. Nat Genet 25: 25-29, 2000.

17. The Gene Ontology Consortium: Expansion of the gene ontology knowledgebase and resources. Nucleic Acids Res 45: D331-D338, 2017.

18. Kanehisa M, Furumichi M, Tanabe M, Sato Y and Morishima K: KEGG: New perspectives on genomes, pathways, diseases and drugs. Nucleic Acids Res 45: D353-D361, 2017.

19. Szklarczyk D, Morris JH, Cook H, Kuhn M, Wyder S, Simonovic M, Santos A, Doncheva NT, Roth A, Bork P, et al: The STRING database in 2017: Quality-controlled protein-protein association networks, made broadly accessible. Nucleic Acids Res 45: D362-D368, 2017.

20. Gao F, Liang H, Lu H, Wang J, Xia M, Yuan Z, Yao Y, Wang T, Tan X, Laurence A, et al: Global analysis of DNA methylation in hepatocellular carcinoma by a liquid hybridization capture-based bisulfite sequencing approach. Clin Epigenetics 7: 86, 2015.

21. Barrett T, Wilhite SE, Ledoux P, Evangelista C, Kim IF, Tomashevsky M, Marshall KA, Phillippy KH, Sherman PM, Holko M, et al: NCBI GEO: Archive for functional genomics data sets-Update. Nucleic Acids Res 41 (Database Issue): D991-D995, 2013.

22. Anaya J: OncoLnc: Linking TCGA survival data to mRNAs, miRNAs and lncRNAs. PeerJ Comput Sci 2: e67, 2016.

23. Kulis $M$ and Esteller M: 2-DNA methylation and cancer In: Epigenetics and Cancer, Part A. vol 70 Herceg Z and Ushijima TBT-A in G (eds.) Academic Press, pp27-56, 2010.

24. Sato Y, Yoshizato T, Shiraishi Y, Maekawa S, Okuno Y, Kamura T, Shimamura T, Sato-Otsubo A, Nagae G, Suzuki H, et al: Integrated molecular analysis of clear-cell renal cell carcinoma. Nat Genet 45: 860-867, 2013.

25. Wang C, Mckeithan TW, Gong Q, Zhang W, Bouska A, Rosenwald A, Gascoyne RD, Wu X, Wang J, Muhammad Z, et al: IDH2R172 mutations define a unique subgroup of patients with angioimmunoblastic T-cell lymphoma. Blood 126: 1741-1752, 2015.

26. Betti M, Aspesi A, Biasi A, Casalone E, Ferrante D, Ogliara P, Gironi LC, Giorgione R, Farinelli P, Grosso F, et al: CDKN2A and BAP1 germline mutations predispose to melanoma and mesothelioma. Cancer Lett 378: 120-130, 2016

27. Nome T, Hoff AM, Bakken AC, Rognum TO, Nesbakken A and Skotheim RI: High frequency of fusion transcripts involving TCF7L2 in colorectal cancer: Novel fusion partner and splice. PLoS One 9: e91264, 2014.

28. Inamoto S, Itatani $\mathrm{Y}$, Yamamoto $\mathrm{T}$, Minamiguchi $\mathrm{S}$, Hirai $\mathrm{H}$, Iwamoto M, Hasegawa S, Taketo MM, Sakai Y and Kawada K Loss of SMAD4 promotes colorectal cancer progression by accumulation of myeloid-derived suppressor cells through the CCL15-CCR1 chemokine axis. Clin Cancer Res 22: 492-501, 2016.
29. Mura M, Hopkins TG, Michael T, Abd-Latip N, Weir J, Aboagye E, Mauri F, Jameson C, Sturge J, Gabra H, et al: LARP1 post-transcriptionally regulates mTOR and contributes to cancer progression. Oncogene 34: 5025-5036, 2015.

30. Paik SS, Jang KS, Song YS, Jang SH, Min KW, Han HX, Na W, Lee KH, Choi D and Jang SJ: Reduced expression of Apaf-1 in colorectal adenocarcinoma correlates with tumor progression and aggressive phenotype. Ann Surg Oncol 14: 3453-3459, 2007.

31. Christoph F, Kempkensteffen C, Weikert S, Köllermann J, Krause H, Miller K, Schostak M and Schrader M: Methylation of tumour suppressor genes APAF-1 and DAPK-1 and in vitro effects of demethylating agents in bladder and kidney cancer. $\mathrm{Br}$ J Cancer 95: 1701-1707, 2006

32. Ishizawa J, Kojima K, Hail N Jr, Tabe Y and Andreeff M: Expression, function, and targeting of the nuclear exporter chromosome region maintenance 1 (CRM1) protein. Pharmacol Ther 153: 25-35, 2015 .

33. Yoshimura M,Ishizawa J, Ruvolo V, Dilip A, Quintás-Cardama A, McDonnell TJ, Neelapu SS, Kwak LW, Shacham S, Kauffman M, et al: Induction of p53-mediated transcription and apoptosis by exportin-1 (XPO1) inhibition in mantle cell lymphoma. Cancer Sci 105: 795-801, 2014.

34. Gao W, Lu C, Chen L and Keohavong P: Overexpression of CRM1: A characteristic feature in a transformed phenotype of lung carcinogenesis and a molecular target for lung cancer adjuvant therapy. J Thorac Oncol 10: 815-825, 2015.

35. Zhou F, Qiu W, Yao R, Xiang J, Sun X, Liu S, Lv J and Yue L: CRM1 is a novel independent prognostic factor for the poor prognosis of gastric carcinomas. Med Oncol 30: 726, 2013.

36. Kojima K, Kornblau SM, Ruvolo V, Dilip A, Duvvuri S, Davis RE, Zhang M, Wang Z, Coombes KR, Zhang N, et al: Prognostic impact and targeting of CRM1 in acute myeloid leukemia. Blood 121: 4166-4174, 2013.

37. Huang WY, Yue L, Qiu WS, Wang LW, Zhou XH and Sun YJ: Prognostic value of CRM1 in pancreas cancer. Clin Invest Med 32: E315, 2009.

38. Cairns RA, Iqbal J, Lemonnier F, Kucuk C, de Leval L, Jais JP, Parrens M, Martin A, Xerri L, Brousset P, et al: IDH2 mutations are frequent in angioimmunoblastic T-cell lymphoma. Blood 119: 1901-1903, 2012.

39. Koh J, Cho H, Kim H, Kim SI, Yun S, Park CK, Lee SH, Choi SH and Park SH: IDH2 mutation in gliomas including novel mutation. Neuropathology 35: 236-244, 2015.

40. Green CL, Evans CM, Zhao L, Hills RK, Burnett AK, Linch DC and Gale RE: The prognostic significance of IDH2 mutations in AML depends on the location of the mutation. Blood 118: 409-412, 2011.

41. Liu WR, Tian MX, Jin L, Yang LX, Ding ZB, Shen YH, Peng YF, Zhou J, Qiu SJ, Dai Z, et al: High expression of 5-hydroxymethylcytosine and isocitrate dehydrogenase 2 is associated with favorable prognosis after curative resection of hepatocellular carcinoma. J Exp Clin Cancer Res 33: 32, 2014.

42. Wang XW, Ciccarino P, Rossetto M, Boisselier B, Marie Y, Desestret V, Gleize V, Mokhtari K, Sanson M and Labussière M: IDH mutations: Genotype-phenotype correlation and prognostic impact. Biomed Res Int 2014: 540236, 2014.

This work is licensed under a Creative Commons Attribution-NonCommercial-NoDerivatives 4.0 International (CC BY-NC-ND 4.0) License. 